\title{
Online Ciphers from Tweakable Blockciphers
}

\author{
Phillip Rogaway and Haibin Zhang \\ Dept. of Computer Science, University of California, Davis, California 95616, USA \\ \{rogaway, hbzhang\}@cs.ucdavis.edu
}

\begin{abstract}
Online ciphers are deterministic length-preserving permutations $\mathcal{E}_{K}:\left(\{0,1\}^{n}\right)^{+} \rightarrow\left(\{0,1\}^{n}\right)^{+}$where the $i$-th block of ciphertext depends only on the first $i$ blocks of plaintext. Definitions, constructions, and applications for these objects were first given by Bellare, Boldyreva, Knudsen, and Namprempre. We simplify and generalize their work, showing that online ciphers are rather trivially constructed from tweakable blockciphers, a notion of Liskov, Rivest, and Wagner. We go on to show how to define and achieve online ciphers for settings in which messages need not be a multiple of $n$ bits.
\end{abstract}

Key words: Online ciphers, modes of operation, provable security, symmetric encryption, tweakable blockciphers.

\section{Introduction}

BACKGROUND. Informally, a cryptographic transform is said to be online if it can be computed by an algorithm that reads in the (unknown number of) input bits - in order, one at a time - as it writes out the corresponding output bits - again in order, one at a time - never using more than a constant amount of memory or incurring more than a constant amount of latency. ${ }^{1}$ Most blockcipher modes of operation are online - for example, modes like CBC, HMAC, and GCM certainly are. But one kind of transformation is not online, and can never be online: a general cipher [20], one secure in the customary sense of a PRP (a pseudorandom permutation). Such objects take a key $K$ and a plaintext $M$ of unbounded length and produce a ciphertext $C$ of length $|M|$, doing so in such a way that the mapping resembles a random permutation. EME2 is a soon-tobe-standardized example [14]. The reason an online cipher can't be PRP-secure is simple: the first bit of output, for example, has got to depend on every bit of input, else it is trivial to distinguish the cipher from a random permutation. This requirement makes bounded memory, or latency, an impossibility.

One can weaken PRP security to capture what is possible in the online setting. Bellare, Boldyreva, Knudsen, and Namprempre (BBKN) were the first to do so, defining online ciphers [3]. The authors fix a parameter $n$ (likely the blocksize of some underlying blockcipher). They then demand that the $i$-th $(n$ bit) block of ciphertext depend only on the first $i$ blocks of plaintext (and, of

\footnotetext{
${ }^{1}$ For online ciphers, two alternative formulations - one corresponding to the opening sentence of the abstract, plus one other - will subsequently be described.
} 
course, the key). ${ }^{2}$ BBKN did not explicitly demand that encryption and decryption be computable with constant memory and latency, but follow-on work by Boldyreva and Taesombut strengthened BBKN's definition in a way that ensures this is so [9].

Like other kinds of ciphers, online ciphers can be secure in either the CPA (chosen plaintext) or CCA (chosen ciphertext, or "strong") sense, depending on whether the adversary is given oracle access to the decryption (or "backwards") functionality as well as the encryption (or "forwards") functionality. For online ciphers it initially seemed as though CCA-security was harder to achieve than CPA-security [3], but, at present, the most efficient CCA-secure construction has essentially the same overhead as the most efficient CPA-secure one, needing just one extra xor per block [22].

Online ciphers are useful tools. For example, BBKN demonstrate a simple recipe for turning a CCA-secure online cipher into an authenticated-encryption scheme (one prepends and appends a random value $R \in\{0,1\}^{n}$ ) [4]; Boldyreva and Taesombut (following, Fouque Joux, Martinet, and Valette [10]) show how to turn a CCA-secure online cipher into either an online encryption scheme secure against "blockwise-adaptive" CCA attacks, or else an online authenticatedencryption (AE) scheme likewise secure against BA-CCA attacks [9]; and Amanatidis, Boldyreva, and O'Neill describe the use of online ciphers to solve a database-security problem [1].

Our CONTRIBution. In this paper we make two contributions. First, we recast the constructions of BBKN [3,4], plus a subsequent construction by Nandi [21, 22], into the language of tweakable blockciphers, a notion of Liskov, Rivest, and Wagner [17]. The new starting point yields constructions more general and transparent than those before. See Fig. 1. Second, we show how to relax the notion of an online cipher to deal with messages that are not a multiple of $n$ bits. Besides definitions, we provide a simple and efficient construction to handle this setting. Dealing with arbitrary-length inputs is a necessary precursor to practical schemes, which we also describe.

Discussion. The original BBKN paper had fairly complex schemes and proofs [3]. Nandi found some bugs in these proofs and offered up his own [21,22]. BBKN corrected the issues in their proofs, which they regarded as minor, but the proofs remain complex [4]. BBKN's modes relied on xor-universal hash functions, and subsequent work did too, or else doubled the number of blockcipher calls $[3,4,21,22]$. Our own constructions are simple, and they are natural generalizations of the existing schemes. The proofs are simple too. We do not regard this simplicity as a defect. Without the tweakable-blockcipher abstraction, constructions and proofs in this domain are not simple, as the above history suggests.

The question of fractional final blocks was earlier asked by Nandi [22, p. 361]. Note that one cannot just say to pad to the next multiple of $n$ (as suggested, for

${ }^{2}$ It follows that the $i$-th block of plaintext will likewise depend only on the key and the first $i$ blocks of ciphertext. 
Joux, Martinet, and Valette [15] implicitly argue that our notion of security for online ciphers is not strong enough, since it treats the encryption operation as "atomic," not attending to attacks that, for example, select the second block of a plaintext being encrypted based on the encryption of the first. We do not dispute this insight, but nonetheless prefer not to deal with these blockwise-adaptive adversarial attacks. First, enriching the security notion to allow for them hardly changes things in the CPA-setting [11, Theorem 8]. Second, "atomic" online ciphers are already useful for higher-level applications, as described above. Finally, "true" CCA security becomes impossible in the BA setting, leading to more subtle definitions for what actually is achievable [9].

\section{Preliminaries}

Notation. A string is a member of $\{0,1\}^{*}$. The notation $A \| B$, or just $A B$, denotes the concatenation of strings $A$ and $B$. If $X$ is a string then $|X|$ denotes its length. The empty string is denoted $\varepsilon$. Throughout this paper we fix a positive number $n$ called the blocksize. The set $\left(\{0,1\}^{n}\right)^{+}$is the set of all strings having length $j n$ for some $j \geq 1$. If $X \in\left(\{0,1\}^{n}\right)^{+}$we let $X[i]$ denotes its $i$ th $n$-bit block, so $X=X[1] \cdots X[m]$ where $m=|X| / n$. We will later extend this notation to the case when $X$ is not a multiple of $n$ bits. We write $X[i . . j]$ for $X[i] \cdots X[j]$.

Ciphers. A map $f: \mathcal{X} \rightarrow \mathcal{X}$ for $\mathcal{X} \subseteq\{0,1\}^{*}$ is a length-preserving function if $|f(x)|=|x|$ for all $x \in\{0,1\}^{*}$. It is a length-preserving permutation if it is also a permutation. A cipher is a map $\widetilde{E}: \mathcal{K} \times \mathcal{M} \rightarrow \mathcal{M}$ where $\mathcal{K}$ is a nonempty set (finite or otherwise endowed with some distribution), $\mathcal{M} \subseteq\{0,1\}^{*}$ is a nonempty set, and $\mathcal{E}_{K}=\mathcal{E}(K, \cdot)$ is a length-preserving permutation for all $K \in \mathcal{K}$. The set $\mathcal{K}$ is called the key space and $\mathcal{M}$ is called the message space. If $\mathcal{E}: \mathcal{K} \times \mathcal{M} \rightarrow$ $\mathcal{M}$ is a cipher then its inverse is the cipher $\mathcal{E}^{-1}: \mathcal{K} \times \mathcal{M} \rightarrow \mathcal{M}$ defined by $\mathcal{E}^{-1}(K, Y)=\mathcal{E}_{K}^{-1}(Y)$ being the unique point $X$ such that $\mathcal{E}_{K}(X)=Y$.

BlockCIPHERS AND TWEAKABle BLOCKCIPHERS. A blockcipher is a function $E: \mathcal{K} \times\{0,1\}^{n} \rightarrow\{0,1\}^{n}$ where $\mathcal{K}$ is a finite nonempty set and $E_{K}(\cdot)=E(K, \cdot)$ is a permutation on $\{0,1\}^{n}$ for every $K \in \mathcal{K}$. Equivalently, a blockcipher is a cipher with message space $\mathcal{M}=\{0,1\}^{n}$. A tweakable blockcipher is a function $\widetilde{E}: \mathcal{K} \times \mathcal{T} \times\{0,1\}^{n} \rightarrow\{0,1\}^{n}$ where $\mathcal{K}$ is a finite nonempty set and $\mathcal{T}$ is a nonempty set (the tweak space) and $\widetilde{E}_{K}^{T}(\cdot)=\widetilde{E}(K, T, \cdot)$ is a permutation on $\{0,1\}^{n}$ for every $K \in \mathcal{K}, T \in \mathcal{T}$.

Let $\operatorname{Perm}(n)$ be the set of all permutations on $n$ bits, $\operatorname{Perm}(\mathcal{M})$ be the set of all length-preserving permutations on the finite set $\mathcal{M} \subseteq\{0,1\}^{*}$, and $\operatorname{Perm}(\mathcal{T}, n)$ the set of all functions $\pi: \mathcal{T} \times\{0,1\}^{n} \rightarrow\{0,1\}^{n}$ where $\pi_{T}(\cdot)=\pi(T, \cdot)$ is a permutation for each $T \in \mathcal{T}$. We may regard $\operatorname{Perm}(n), \operatorname{Perm}(\mathcal{M})$, and $\operatorname{Perm}(\mathcal{T}, n)$ as blockciphers, ciphers, and tweakable blockciphers, respectively; they are the ideal blockcipher on $n$ bits, the ideal cipher on $\mathcal{M}$, and the ideal tweakable blockcipher on $n$ bits and tweak space $\mathcal{T}$. When an adversary $\mathcal{A}$ is run with an oracle $\mathcal{O}$ we let $\mathcal{A}^{\mathcal{O}} \Rightarrow 1$ denote the event that $\mathcal{A}$ outputs 1 . Define 
the prp, \pm prp, $\widetilde{\operatorname{prp}}$, and $\pm \widetilde{\operatorname{prp}}$ advantage of $\mathcal{A}$ against $E$ or $\widetilde{E}$ by:

$$
\begin{aligned}
& \operatorname{Adv}_{E}^{\operatorname{prp}}(\mathcal{A})=\operatorname{Pr}\left[K \stackrel{\&}{\leftarrow} \mathcal{K}: \mathcal{A}^{E_{K}} \Rightarrow 1\right]-\operatorname{Pr}\left[\pi \stackrel{\&}{\leftarrow} \operatorname{Perm}(n): \mathcal{A}^{\pi} \Rightarrow 1\right] \\
& \operatorname{Adv}_{E}^{ \pm \operatorname{prp}}(\mathcal{A})=\operatorname{Pr}\left[K \stackrel{\&}{\leftarrow} \mathcal{K}: \mathcal{A}^{E_{K}, E_{K}^{-1}} \Rightarrow 1\right]-\operatorname{Pr}\left[\pi \stackrel{\$}{\leftarrow} \operatorname{Perm}(n): \mathcal{A}^{\pi, \pi^{-1}} \Rightarrow 1\right] \\
& \operatorname{Adv}_{\widetilde{E}}^{\overparen{\operatorname{prp}}}(\mathcal{A})=\operatorname{Pr}\left[K \stackrel{\$}{\leftarrow} \mathcal{K}: \mathcal{A}^{\widetilde{E}_{K}} \Rightarrow 1\right]-\operatorname{Pr}\left[\pi \stackrel{\$}{\leftarrow} \operatorname{Perm}(\mathcal{T}, n): \mathcal{A}^{\pi} \Rightarrow 1\right]
\end{aligned}
$$

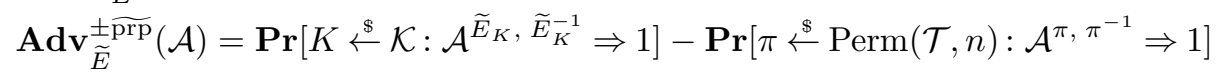

ONLINE CIPHERS. A length-preserving function $f:\left(\{0,1\}^{n}\right)^{+} \rightarrow\left(\{0,1\}^{n}\right)^{+}$is online if, for all $i, f(X)[1 . . i]$ depends only on $X[1 . . i]$. Here we say that $f(X)[1 . . i]$ depends only on $X[1 . . i]$ if $f(X Y)[1 . . i]=f\left(X Y^{\prime}\right)[1 . . i]$ for all $X \in\{0,1\}^{i n}$ and $Y, Y^{\prime} \in\{0,1\}^{*}$ where $f(X Y)$ and $f\left(X Y^{\prime}\right)$ are defined. A cipher $\mathcal{E}: \mathcal{K} \times$ $\left(\{0,1\}^{n}\right)^{+} \rightarrow\left(\{0,1\}^{n}\right)^{+}$is online if each $\mathcal{E}_{K}$ is. Let Online $(n): \mathcal{K} \times\left(\{0,1\}^{n}\right)^{+} \rightarrow$ $\left(\{0,1\}^{n}\right)^{+}$be the ideal online cipher on $n$ bits: each key names one of the possible online ciphers, the set being given the uniform distribution in the natural way. If $\mathcal{E}: \mathcal{K} \times\left(\{0,1\}^{n}\right)^{+} \rightarrow\left(\{0,1\}^{n}\right)^{+}$is an online cipher and $\mathcal{A}$ is an adversary we define:

$$
\begin{aligned}
\operatorname{Adv}_{\mathcal{E}}^{\text {oprp }}(\mathcal{A}) & =\operatorname{Pr}\left[K \stackrel{\&}{\leftarrow} \mathcal{K}: \mathcal{A}^{\mathcal{E}_{K}} \Rightarrow 1\right]-\operatorname{Pr}\left[\pi \stackrel{\$}{\leftarrow} \operatorname{Online}(n): \mathcal{A}^{\pi} \Rightarrow 1\right] \\
\operatorname{Adv}_{\mathcal{E}}^{ \pm \operatorname{oprp}}(\mathcal{A}) & =\operatorname{Pr}\left[K \stackrel{\$}{\leftarrow} \mathcal{K}: \mathcal{A}^{\mathcal{E}_{K}, \mathcal{E}_{K}^{-1}} \Rightarrow 1\right]-\operatorname{Pr}\left[\pi \stackrel{\&}{\leftarrow} \operatorname{Online}(n): \mathcal{A}^{\pi, \pi^{-1}} \Rightarrow 1\right]
\end{aligned}
$$

We comment that the definitions allow variable-input-length (VIL) attacks: the adversary may ask queries of varying lengths. On the other hand, definitions only countenance ciphers on $\mathcal{M}=\left(\{0,1\}^{n}\right)^{+}$, and it is not obvious what to do beyond this domain, Online $(n)$ being quite specific to it.

Discussion. The notion for an online cipher just given, taken from BBKN [3], can be criticized for not prohibiting, for example, that that computation of $C[m]$ requires one to retain all of $M[1] \cdots M[m]$. A stronger notion appears in Boldyreva and Taesombut [9], the definition asserting that $C[i]$ may only depend on $M[i], M[i-1], C[i-1]$, and the underlying key. We believe that this requirement does not make for a desirable security definition: the cipher in which each $C[i]$ is a random permutation of $M[i]$, tweaked by $M[i-1] \| C[i-1]$, ought not to be regarded ideal, since one can easily do better and still, intuitively, be "online." Still, our constructions enjoy the BT-style locality property, ensuring that they can be implemented with constant latency and memory.

An alternative notion for an online cipher would capture the intuition from the opening paragraph of this paper, saying that a cipher $\mathcal{E}: \mathcal{K} \times \mathcal{M} \rightarrow \mathcal{M}$ is Online $[m]$ if it can be implemented by an algorithm that is fed in bits one at a time, and that retains just $m$ bits of state. This would natively handle ciphers on arbitrary bit strings. We leave it as an open question to explore these ideas.

\section{Online Ciphers Achieving CPA-Security}

Let $\widetilde{E}: \mathcal{K} \times\{0,1\}^{n} \times\{0,1\}^{n} \rightarrow\{0,1\}^{n}$ be a tweakable blockcipher. From this primitive we define a cipher $\mathcal{E}=\mathrm{TC} 1[\widetilde{E}]$ with key space $\mathcal{K}$ and message space 
$\mathcal{M}=\left(\{0,1\}^{n}\right)^{+}$. See Fig. 1. The construction is online CPA-secure, as formalized below.

Theorem 1 (TC1 is oprp-secure). Let $\widetilde{\pi}=\operatorname{Perm}\left(\{0,1\}^{n}, n\right)$. If $\mathcal{A}$ asks queries having at most $\sigma$ blocks then $\operatorname{Adv}_{\mathrm{TC} 1[\widetilde{\pi}]}^{\text {oprp }}(\mathcal{A}) \leq 1.5 \sigma^{2} / 2^{n}$.

We omit the proof because we will in a moment be proving, by analogous but slightly more involved means, what is essentially a stronger result: online CCAsecurity for the equally efficient cipher TC3. The complexity-theoretic analog for the theorem, using a "real" tweakable PRP $\widetilde{E}$ instead of the ideal tweakable $\operatorname{PRP} \widetilde{\pi}$, follows by standard techniques. One would need $\widetilde{E}$ to be secure in the prp-sense. We omit the theorem statement, showing later how it would look for scheme TC3.

Mechanism TC1 is a generalization of BBKN's mode of operation HCBC1 [4] (formerly named HCBC [3]); the latter can be realized as a special case of TC1 by selecting the tweakable blockcipher $\widetilde{E}:\left(\mathcal{K}_{1} \times \mathcal{K}_{2}\right) \times\{0,1\}^{n} \rightarrow\{0,1\}^{n}$ to be $\widetilde{E}_{K 1 K 2}^{T}(X)=E_{K 1}\left(M \oplus H_{K 2}(T)\right)$ where $H: \mathcal{K}_{2} \times\{0,1\}^{n} \rightarrow\{0,1\}^{n}$ is an almost-xor universal hash function and $E$ is a blockcipher. ${ }^{3}$ This is in fact the "standard" construction of a tweakable blockcipher from an ordinary one [17]. Of course one can instantiate the tweakable blockcipher $\widetilde{E}$ from an ordinary blockcipher $E$ in a variety of other ways as well. We comment that TC1 can also be regarded as Liskov, Rivest, and Wagner's "tweak block chaining" mode [17, Section 4] but with a zero IV.

Note that TC1 is not a secure online cipher with respect to CCA attacks. A simple attack is as follows. The adversary makes a decryption query of $C\|C\| C$ for any $C \in\{0,1\}^{n}$. The oracle returns $M_{1}\left\|M_{2}\right\| M_{3}$ as the reply. If $M_{2}=M_{3}$, return 1 ; otherwise, return 0 . Under the TC1 construction, one will always have that $M_{2}=M_{3}$, but with a random on-line cipher this will rarely be true.

\section{Online Ciphers Achieving CCA-Security}

Let $\widetilde{E}: \mathcal{K} \times\{0,1\}^{2 n} \times\{0,1\}^{n} \rightarrow\{0,1\}^{n}$ be a tweakable blockcipher. From this primitive we define a cipher $\mathcal{E}=\mathrm{TC} 2[\widetilde{E}]$ with key space $\mathcal{K}$ and message space $\mathcal{M}=\left(\{0,1\}^{n}\right)^{+}$. Again see Fig. 1. The construction is CCA-secure, as formalized below.

Theorem 2 (TC2 is \pm oprp-secure). Let $\widetilde{\pi}=\operatorname{Perm}\left(\{0,1\}^{2 n}, n\right)$. If $\mathcal{A}$ asks queries having at most $\sigma$ blocks then $\operatorname{Adv}_{\mathrm{TC} 2[\tilde{\pi}]}^{ \pm \mathrm{oprp}}(\mathcal{A}) \leq 1.5 \sigma^{2} / 2^{n}$.

We again omit the proof, and the complexity-theoretic analog, which would this time need the $\pm \widehat{\text { prp }}$ assumption, preferring, for concision, to do this just for TC2's more efficient cousin, TC3.

\footnotetext{
${ }^{3}$ We recall the definition, due to Krawczyk [16], that $H: \mathcal{K} 2 \times \mathcal{X} \rightarrow\{0,1\}^{n}$ is $\epsilon$-almost XOR universal $\left(\epsilon\right.$-AXU) if for all distinct $X, X^{\prime} \in \mathcal{X}$ and all $C \in\{0,1\}^{n}$ we have that $\operatorname{Pr}\left[H_{K}(X) \oplus H_{K}\left(X^{\prime}\right)=C\right] \leq \epsilon$, the probability over $K \stackrel{\$}{\leftarrow} \mathcal{K} 2$. Simple constructions achieve $\epsilon=2^{-n}$, the minimum value possible.
} 
Mechanism TC2 is a generalization of BBKN's mode HCBC2 [4] (formerly named HPCBC), which can be regarded as TC2 with a tweakable blockcipher $\widetilde{E}:\left(\mathcal{K}_{1} \times \mathcal{K}_{2}\right) \times\{0,1\}^{n} \rightarrow\{0,1\}^{n}$ of $\widetilde{E}_{K 1 K 2}^{T}(X)=E_{K 1}\left(M \oplus H_{K 2}(T)\right) \oplus H_{K 2}(T)$ where $H: \mathcal{K}_{2} \times\{0,1\}^{2 n} \rightarrow\{0,1\}^{n}$ is an almost-xor universal hash function and $E$ is a blockcipher. This is also the "standard" construction of a strong tweakable blockcipher from an ordinary one [17].

We are now ready to consider TC3. Let $\widetilde{E}: \mathcal{K} \times\{0,1\}^{n} \times\{0,1\}^{n} \rightarrow\{0,1\}^{n}$ be a tweakable blockcipher. From this primitive define the online cipher $\mathcal{E}=$ TC3 $[\widetilde{E}]$ with key space $\mathcal{K}$ and message space $\mathcal{M}=\left(\{0,1\}^{n}\right)^{+}$. Again see Fig. 1. The construction is CCA-secure, as formalized below.

Theorem 3 (TC3 is \pm oprp-secure). Let $\widetilde{\pi}=\operatorname{Perm}\left(\{0,1\}^{n}, n\right)$. If $\mathcal{A}$ asks queries having at most $\sigma$ blocks then $\operatorname{Adv}_{\mathrm{TC} 3[\tilde{\pi}]}^{ \pm \mathrm{oprp}}(\mathcal{A}) \leq 1.5 \sigma^{2} / 2^{n}$.

The idea of the proof is to "give up" — regard the adversary as having won-if we ever generate a "new" tweak that collides with any prior one.

Proof. Without loss of generality we can assume that $\mathcal{A}$ is deterministic and makes queries totaling exactly $\sigma$ blocks. We can further assume that it never repeats an encryption query, never repeats a decryption query, never asks a decryption query of a value that it earlier received from an encryption query, and never asks an encryption query of a value that it earlier received from a decryption query. For strings $X, X_{1}, \ldots, X_{I} \in\left(\{0,1\}^{n}\right)^{*}$, let find $\left(X ; X_{1}, \ldots, X_{I}\right)$ be the unique pair of numbers $(\imath, \ell)$ for which $X$ and $X_{\imath}$ share a common prefix $X[1 . . \ell]=X_{\imath}[1 . . \ell]$, no $X_{\jmath}(\jmath \in[1 . . I])$ shares a longer common prefix with $X$ $\left(X[1 . . \ell+1]=X_{j}[1 . . \ell+1]\right)$, and $\imath$ is the smallest index in $[1 . . I]$ for which the above is true. If $X=\varepsilon$ define find $\left(X ; X_{1}, \ldots, X_{I}\right)=(0,0)$. By way of examples, if $a, b, c \in\{0,1\}^{n}$ are distinct blocks then find $(a b c a ; a b a a, a b c b, a b c c)=(2,3)$, find $(a b c a ; a, a b c, a b c a b)=(3,4)$, and find $(a b c a ; b b a b, c b a, b)=(1,0)$.

We employ the code-based games [6] shown in Fig. 2. Booleans are silently initialized to false and integers to 0 . The one variable that is a set, $\mathcal{T}$, is silently initialized to $\left\{0^{n}\right\}$. (This is done because $\mathcal{T}$ will be used to record the set of tweaks that have been utilized and, in effect, $0^{n}$ is a tweak that is always usedit is used in processing each query's first block.) Partial functions $\pi_{x}$ (where $\left.x \in\{0,1\}^{*}\right)$ are, initially, everywhere undefined. As they grow we refer to their current domain and range by domain $\left(\pi_{x}\right)$ and range $\left(\pi_{x}\right)$. We write codomain $\left(\pi_{x}\right)$ and corange $\left(\pi_{x}\right)$ for the complements relative to $\{0,1\}^{n}$.

We begin Fig. 2 with game $G_{1}$, which precisely emulates the TC3 construction with the ideal tweakable blockcipher $\widetilde{E}$. We end with game $G_{6}$, which precisely emulates the ideal online cipher. Thus we have that $\operatorname{Adv}_{\widetilde{E}}^{\text {oprp }}(\mathcal{A})=$ $\operatorname{Pr}\left[G_{1}^{\mathcal{A}} \Rightarrow 1\right]-\operatorname{Pr}\left[G_{6}^{\mathcal{A}} \Rightarrow 1\right]$. Games $G_{2}, G_{3}, G_{4}$, and $G_{5}$ are hybrid games in between these two extremes, and we bound the $\operatorname{Pr}\left[G_{1}^{\mathcal{A}} \Rightarrow 1\right]-\operatorname{Pr}\left[G_{6}^{\mathcal{A}} \Rightarrow 1\right]$ as $\sum_{1 \leq j \leq 5}\left(\operatorname{Pr}\left[G_{j}^{\mathcal{A}} \Rightarrow 1\right]-\operatorname{Pr}\left[G_{j+1}^{\mathcal{A}} \Rightarrow 1\right]\right)$.

Passing from games $G_{1}$ to $G_{2}$ is just the usual approach of lazy sampling [6]; the games $G_{1}$ and $G_{2}$ are adversarially indistinguishable. By the game-playing lemma, $\operatorname{Pr}\left[G_{2}^{\mathcal{A}} \Rightarrow 1\right]-\operatorname{Pr}\left[G_{3}^{\mathcal{A}} \Rightarrow 1\right]$ is at most the probability that game $\mathcal{A}$ manages 


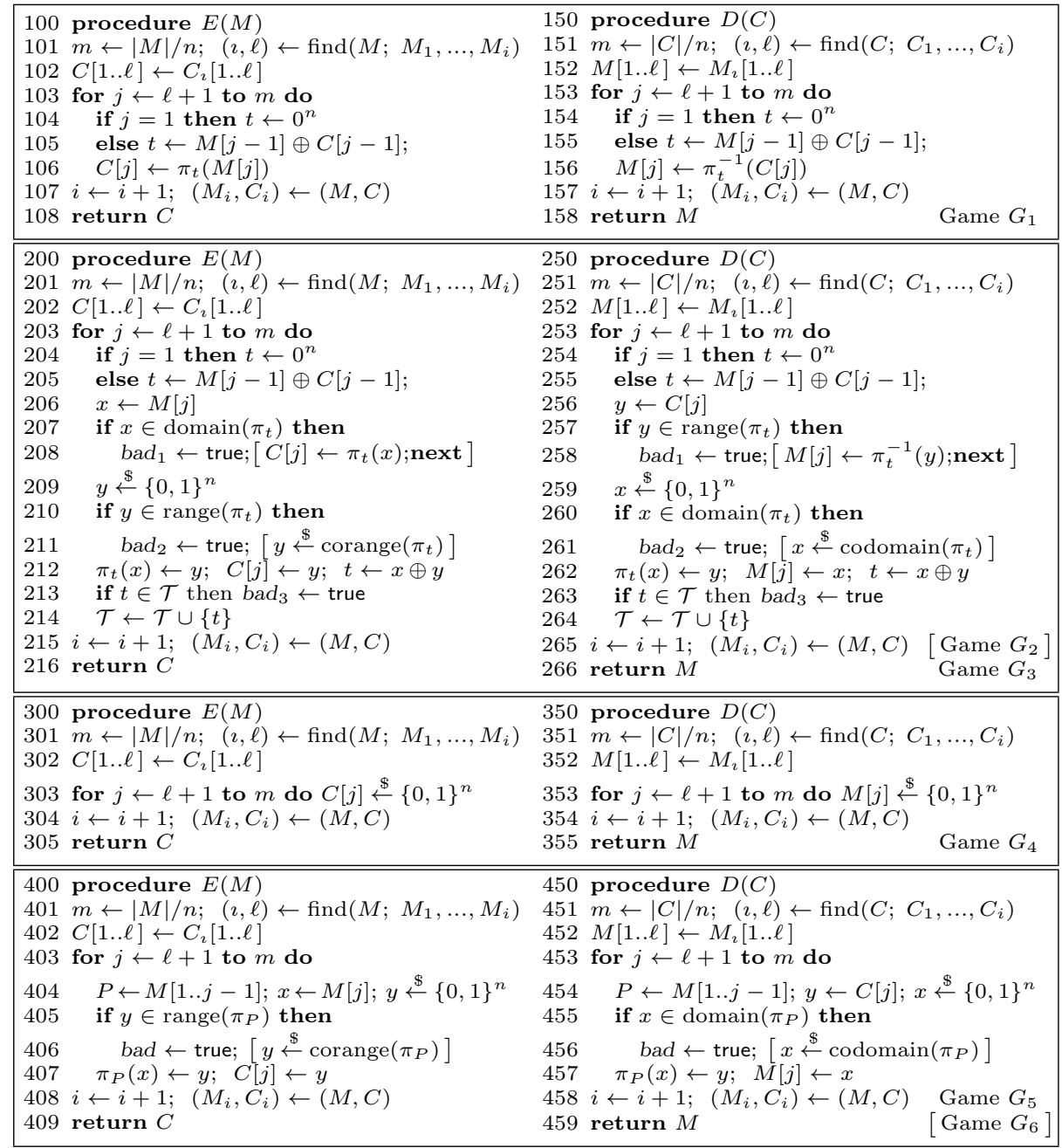

Fig. 2. Games used in the proof of Theorem 3. Game $G_{2}$ includes the bracketed statements while game $G_{3}$ does not. Similarly, game $G_{6}$ includes the bracketed statements while game $G_{5}$ does not. 
to set one of the $\operatorname{bad}_{j}$ variables in game $G_{3}$. The crux of the proof is the following observation:

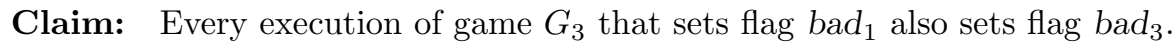

In fact, flag bad $_{3}$ was introduced as a trick for bounding the probability that bad $_{1}$ gets set. The proof of the claim is as follows. Suppose we are executing adversary $\mathcal{A}$ with game $G_{2}$ and, at some point in time it happens that, at line 207, we have $x \in \operatorname{domain}\left(\pi_{t}\right)$, so that $\operatorname{bad}_{1}$ will get set in the following line. Fix the current values $M, m,(\imath, \ell), C[1 . . \ell], t$, and $x$. Now $x$ belonging to $\operatorname{domain}\left(\pi_{t}\right)$ means that some triple $(t, x, y)$ was already added into the set of triples that constitute the partial function $\pi$ (that is, $(t, x, y)$ is "in" $\pi$ if we have set $\left.\pi_{t}(x)=y\right)$. This triple had to have been added to $\pi$ at some earlier execution of line 212 or 262 . We distinguish two possibilities: $(t, x, y)$ is the only triple in $\pi$ with this $t$-value; or else there are, already, at least two distinct triples $(t, x, y),\left(t, x^{\prime}, y^{\prime}\right)$ with this particular $t$-value. In the latter case, when we added the temporally second of these two triples into $\pi$ we checked, at line 213 or 263 , if $t$ was already in $\mathcal{T}$. It would have been, so $\operatorname{bad}_{3}$ would already have been set. What remains is the case that $(t, x, y)$ is the only triple in $\pi$ with the given value $t$. Focus on the fact that $M[1] \cdots M[\ell]$ matches $M_{\imath}[1] \cdots M_{\imath}[\ell]$. Now if the latter string is all of $M_{\imath}$ then there were two prior times that $t$ was generated: one is when $(t, x, y)$ got added to $\pi$, and another is when the final $t$-value was generated in response to the $\imath$-th query - that is, when we executed the final statement at line 212 or 262 . The temporally second of these $t$-producing events would have resulted in production of a $t$ that was already in $\mathcal{T}$ and $\mathrm{bad}_{3}$ would have been set. If, instead, $M[1] \cdots M[\ell]=M_{\imath}[1] \cdots M_{\imath}[\ell]$ and $M_{\imath}$ continues with at least one nonempty block $M_{\imath}[\ell+1]$, then we know that $M[\ell+1] \neq M_{\imath}[\ell+1]$ and the one and only triple in $\pi$ with the given $t$-value must be $\left(t, M_{\imath}[\ell+1], C_{\imath}[\ell+1]\right)$, so $x=M[\ell+1] \neq M_{\imath}[\ell+1]$ could not have caused line 207 to evaluate to true.

The case where bad $_{1}$ gets set on a decryption query, at line 258 , is symmetric with the paragraph above: again bad $_{3}$ will already have been set. This completes the proof of the claim.

Continuing, we now know that $\operatorname{Pr}\left[\operatorname{bad}_{1} \wedge \operatorname{bad}_{2} \wedge \operatorname{bad}_{3}\right]=\operatorname{Pr}\left[\operatorname{bad}_{2} \wedge\right.$ bad $\left._{3}\right]$, which is at most $\operatorname{Pr}\left[\mathrm{bad}_{2}\right]+\operatorname{Pr}\left[\mathrm{bad}_{3}\right]$. The first probability is at most $0.5 \sigma(\sigma-$ $1) / 2^{n}$ and the second is at most $0.5 \sigma(\sigma+1) / 2^{n}$ (recall that $\mathcal{T}$ was initially seeded with a point). We thus have that $\operatorname{Pr}\left[G_{2}^{\mathcal{A}} \Rightarrow 1\right]-\operatorname{Pr}\left[G_{3}^{\mathcal{A}} \Rightarrow 1\right] \leq \sigma^{2} / 2^{n}$.

Games $G_{3}$ and $G_{4}$ are easily seen to be adversarially indistinguishable; we have simply eliminated the pointless code. Games $G_{4}$ and $G_{5}$ are adversarially indistinguishable; here we are introducing using lazy sampling. Passing from $G_{5}$ to $G_{6}$ can be regarded as a form of the PRP/PRF switching lemma (cf. [4, Lemma 3.7]) and the probability that bad gets set to true in game $G_{6}$ is at most $0.5 \sigma^{2} / 2^{n}$. The theorem now follows.

The complexity-theoretic analog easily follows. This time, we show how the theorem looks. 
Corollary 1 (TC3 is \pm oprp-secure). Let $\widetilde{E}: \mathcal{K} \times\{0,1\}^{n} \times\{0,1\}^{n} \rightarrow\{0,1\}^{n}$ be a tweakable blockcipher. Let $\mathcal{A}$ be an adversary that runs in time $t$ and asks queries totaling at most $\sigma$ blocks. Then there exists an adversary $\mathcal{B}$ such that $\mathbf{A} \mathbf{d} \mathbf{v}_{\mathrm{TC} 3[\widetilde{E}]}^{\mathrm{prp}}(\mathcal{B}) \geq \mathbf{A d v}_{\mathcal{E}}^{\mathrm{oprp}}(\mathcal{A})-1.5 \sigma^{2} / 2^{n}$. Adversary $\mathcal{B}$ runs in time at most $t+$ cn $\sigma$, for some absolute constant $c$, and asks at most $\sigma$ queries.

Mode TC3, beyond being a natural simplification to the generalization to mode $\mathrm{HCBC} 2$ [4], is a generalization of Nandi's mode MHCBC [22]; the latter can be realized as a special case of TC3 by selecting the tweakable blockcipher $\widetilde{E}:\left(\mathcal{K}_{1} \times\right.$ $\left.\mathcal{K}_{2}\right) \times\{0,1\}^{n} \rightarrow\{0,1\}^{n}$ to be $\widetilde{E}_{K 1 K 2}^{T}(X)=E_{K 1}\left(M \oplus H_{K 2}(T)\right) \oplus H_{K 2}(T)$ where $H: \mathcal{K}_{2} \times\{0,1\}^{n} \rightarrow\{0,1\}^{n}$ is an almost-xor universal hash function and $E$ is a blockcipher [17].

\section{Online Ciphers for Arbitrary-Length Strings}

We start out by extending the notation $M[i]$ so that, for each nonempty string $M$ we have that $M=M[1] \cdots M[m-1] M[m]$ where $m=\lfloor|M| / n\rfloor,|M[i]|=n$ for all $1 \leq i \leq m-1$, and $n \leq|M[m]| \leq 2 n-1$. In other words, when $M$ is not a multiple of $n$ bits its final block $M[m]$ is chosen to be long, having between $n+1$ and $2 n-1$ bits. All other blocks remain $n$-bits in length. With this notation in hand we define Online ${ }^{*}(n)$ to be the set of all length-preserving permutations $\pi:\{0,1\}^{*} \rightarrow\{0,1\}^{*}$ such that $\pi(M[1] \cdots M[i])$ depends only on $M[1] \cdots M[i]$ (for all $i \geq 1$ ). This set can be regarded as an idealized cipher, just like $\operatorname{Perm}(n)$ and Online $(n)$. Now if $\mathcal{E}: \mathcal{K} \times\{0,1\}^{*} \rightarrow\{0,1\}^{*}$ is an online cipher and $\mathcal{A}$ is an adversary we can extend our prior definitions by using Online* $(n)$ in our reference experiment:

$$
\begin{aligned}
\operatorname{Adv}_{\mathcal{E}}^{\text {oprp }}(\mathcal{A}) & =\operatorname{Pr}\left[K \stackrel{\$}{\leftarrow} \mathcal{K}: \mathcal{A}^{\mathcal{E}_{K}} \Rightarrow 1\right]-\operatorname{Pr}\left[\pi \stackrel{\$}{\leftarrow} \operatorname{Online}^{*}(n): \mathcal{A}^{\pi} \Rightarrow 1\right] \\
\operatorname{Adv}_{\mathcal{E}}^{ \pm \operatorname{oprp}}(\mathcal{A}) & =\operatorname{Pr}\left[K \stackrel{\$}{\leftarrow} \mathcal{K}: \mathcal{A}^{\mathcal{E}_{K}, \mathcal{E}_{K}^{-1}} \Rightarrow 1\right]-\operatorname{Pr}\left[\pi \stackrel{\&}{\leftarrow} \operatorname{Online}^{*}(n): \mathcal{A}^{\pi, \pi^{-1}} \Rightarrow 1\right]
\end{aligned}
$$

There is an alternative notion of security where, when $M$ is not a multiple of $n$ bits, the final block is short (having 1 to $n-1$ bits) instead of long (having $n+1$ to $2 n-1$ bits). There are problems with this alternative notion. First, it is too weak. If the adversary learns $C=E_{K}(X \| 0)$, where $X \in\left(\{0,1\}^{n}\right)^{+}$, then it also knows $C^{\prime}=E_{K}(X \| 1)$, which is just $C$ with its final bit flipped. Second, despite this alternative notion being weak, instantiations are hard. This is because it is not known how to construct from an $n$-bit blockcipher an efficient and provablysecure cipher, with good bounds, for arbitrary input lengths less than $n$; see the literature on "format-preserving encryption" for a discussion of this problem [5]. While this short-string enciphering problem cannot be avoided if the original message $M$ has fewer than $n$ bits, there is no need to deal with it when $|M|$ has more than $n$ bits, which, in applications, is likely to be most or all the time.

Now turning to constructions, let $\widetilde{E}: \mathcal{K} \times\{0,1\}^{n} \times\{0,1\}^{\leq 2 n-1} \rightarrow\{0,1\}^{\leq 2 n-1}$ be a tweakable cipher. From this primitive define the online cipher $\mathcal{E}=\mathrm{TC}^{*}[\widetilde{E}]$ with key space $\mathcal{K}$ and message space $\mathcal{M}=\{0,1\}^{*}$. See Fig. 3 . The construction is CCA-secure, as formalized below. We will take up in the next section how one constructs a tweakable cipher with message space $\{0,1\}^{\leq 2 n-1}$. 


$$
\begin{array}{|ll}
\hline \text { TC3 }^{*} \\
40 & \text { algorithm } \mathcal{E}_{K}(M) \\
41 & \text { if } M=\varepsilon \text { then return } \varepsilon \\
42 & m \leftarrow\lfloor|M| / n\rfloor ; T \leftarrow 0^{n} \\
43 & \text { for } j \leftarrow 1 \text { to } m-1 \text { do } \\
44 & C[j] \leftarrow \widetilde{E}_{K}^{T}(M[j]) \\
45 & T \leftarrow M[j] \oplus C[j] \\
46 & C[m] \leftarrow \widetilde{E}_{K}^{T}(M[m]) \\
47 & \text { return } C \\
\hline
\end{array}
$$

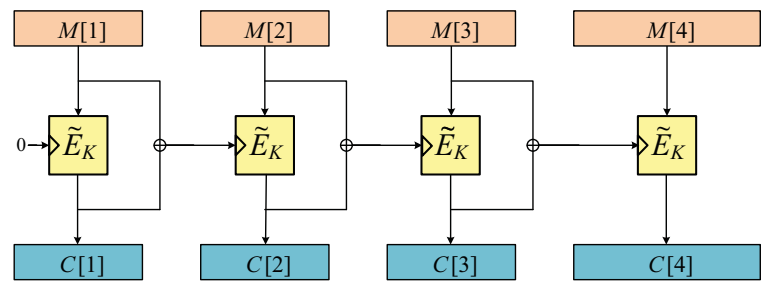

Fig. 3. Mode TC3 ${ }^{*}$. The CCA-secure online cipher now takes an input of arbitrary bit length, but it depends on a richer primitive than does TC3: we start with a cipher $\widetilde{E}:\{0,1\}^{\leq 2 n-1} \rightarrow\{0,1\}^{\leq 2 n-1}$. The block input to $\widetilde{E}$ is "usually" $n$ bits, but a single long-block call (up to $2 n-1$ bits) will be used when $|M| \geq n$ and $n$ doesn't divide $|M|$, while a single short-block call will be needed if $|M|<n$.

Theorem 4 (TC3 $^{*}$ is \pm oprp-secure). Let $\tilde{\pi}=\operatorname{Perm}\left(\{0,1\}^{\leq 2 n-1}\right)$. If $\mathcal{A}$ asks queries having at most $\sigma$ blocks then $\mathbf{A d v}_{\mathrm{TC} 3^{*}[\widetilde{\pi}]}^{ \pm \mathcal{A}}(\mathcal{A}) \leq 1.5 \sigma^{2} / 2^{n}$.

We omit the proof since it is almost the same as that for Theorem 3.

\section{$6 \quad$ Instantiating the Schemes}

Let us consider how to instantiate TC3 starting from a conventional (instead of a tweakable) blockcipher $E:\{0,1\}^{k} \times\{0,1\}^{n} \rightarrow\{0,1\}^{n}$. The simplest and most natural solution is to create the tweakable blockcipher by way of $\widetilde{E}_{K}^{T}(X)=$ $E_{K_{1}}(X \oplus \Delta) \oplus \Delta$ where $\Delta=T \cdot K_{2}$ and $K=K_{1} \| K_{2}$ for $\left|K_{1}\right|=k$ and $\left|K_{2}\right|=n$. Here multiplication, $T \cdot K_{2}$, is in $\mathrm{GF}\left(2^{n}\right)$, representing field points as $n$-bit strings in the usual way. We know that $\widetilde{E}: \mathcal{K} \times\{0,1\}^{n} \times\{0,1\}^{n} \rightarrow\{0,1\}^{n}$ will be a CCA-secure tweakable PRP as long as $E$ is a CCA-secure conventional PRP; this is the well-known construction from Liskov, Rivest, and Wagner [17, Theorem 2], together with the fact that multiplication in $\mathrm{GF}\left(2^{n}\right)$ - that is, $H_{K}(X)=K \cdot X$ is a $2^{-n}$-AXU hash function.

The above construction is quite efficient, involving one blockcipher invocation and one $\mathrm{GF}\left(2^{n}\right)$ multiply for each message block. This is comparable to the work involved with the authenticated-encryption scheme GCM [19], which has, for example, been implemented by Gueron and Kounavis to run as fast as 3.54 cycles per byte [13] (on Intel processors supporting AES and PCLMULQDQ assembly instructions). This timing figure, however, would overestimate the expected speed of TC3, on similar hardware, since the blockcipher chaining in TC3 will decrease instruction-level parallelism.

Following Boldyreva and Taesombut, TC3 can be augmented, with little overhead, to provide a solution to the problem of blockwise-adaptive CCA-secure authenticated-encryption [9]. Doing so would give an AE scheme with efficiency roughly comparable to GCM but provably achieving a useful security property that GCM does not achieve. 
Instantiating TC3* from a conventional blockcipher is more involved than instantiating TC3, as now we need a map $\widetilde{E}: \mathcal{K} \times\{0,1\}^{n} \times\{0,1\}^{\leq 2 n-1} \rightarrow$ $\{0,1\}^{\leq 2 n-1}$. Actually our tweakable cipher will not have to deal with messages having fewer than $n$ bits unless the higher-level construction $\mathcal{E}$ is itself asked to encipher messages of fewer than $n$ bits, so let us put this case aside. Our problem then is to create from an ordinary $n$-bit blockcipher $E$ a VIL-secure tweakable cipher that can encipher messages of $n$ to $2 n-1$ bits. Fortunately there are some ready solutions to this problem. Four or more rounds of Feistel would be the classical approach [18]. One would use a blockcipher-based, tweakdependent round function. A different possibility is the EME2 cipher (formerly named $\mathrm{EME}^{*}$ ) of Halevi [14]; the mechanism was recently approved as the IEEE standard P1619.2-2010. The scheme is simple, provably secure, and, using five blockcipher calls and a modest amount of additional overhead, provides a tweakable and VIL cipher over the domain that we need. More efficient still would be the XLS construction of Ristenpart and Rogaway [24]. This can encipher strings of $n+1$ to $2 n-1$ bits using three blockcipher calls and very little extra work. ${ }^{4}$

Enciphering strings of fewer than $n$ bits takes special techniques. One proposal is FFX [7], which uses a conventional, unbalanced, or alternating Feistel network on these small domains. We note that if one is going to deal with short final blocks by a patchwork of techniques, one for strings in $\{0,1\}^{\leq n-1}$ and one for other strings that are not a multiple of $n$ bits, it is important to use distinct keys, or to use other techniques, to provably ensure VIL security.

\section{Acknowledgments}

Many thanks for the perceptive comments from the anonymous referees. Our apologies that we have not expanded on points where this ought be done.

The authors gratefully acknowledge the support of NSF grant CNS 0904380.

\section{References}

1. G. Amanatidis, A. Boldyreva, and A. O'Neill. Provably-secure schemes for basic query support in outsourced databases. Working Conference on Data and Applications Security (DBSec 2007), LNCS vol. 4602, Springer, pp. 14-30, 2007.

2. G. Bard. A challenging but feasible blockwise-adaptive chosen-plaintext attack on SSL. SECRYPT 2006, International Conference on Security and Cryptography, INSTICC Press, pp. 99-109, 2006.

3. M. Bellare, A. Boldyreva, L. Knudsen, and C. Namprempre. On-line ciphers and the hash-CBC constructions. CRYPTO 2001, LNCS vol. 2139, Springer, pp. 292309, 2001.

\footnotetext{
${ }^{4}$ In fact, none of the methods just surveyed was designed specifically to create a tweakable cipher with $n$-bit tweaks and a message space of $n$ to $2 n-1$ bits. With this limited goal in mind we believe that provably-good methods related to those of Naor-Reingold [23] will give a cipher needing just two blockcipher calls for strings of $n+1$ to $2 n-1$ bits.
} 
4. M. Bellare, A. Boldyreva, L. Knudsen, and C. Namprempre. On-line ciphers and the hash-CBC constructions. Cryptology ePrint report 2007/197, June 29, 2007. Full version of [3].

5. M. Bellare, T. Ristenpart, P. Rogaway, and T. Stegers. Format preserving encryption. Selected Areas in Cryptography, SAC 2009. LNCS vol. 5867, Springer, pp. 295-312, 2009.

6. M. Bellare and P. Rogaway. Code-based game-playing proofs and the security of triple encryption. EUROCRYPT 2006, LNCS vol. 4004, Springer, pp. 409-426, 2006.

7. M. Bellare, P. Rogaway, and T. Spies. The FFX mode of operation for formatpreserving encryption (draft 1.1). NIST submission, February 2010. See also the addendum (September 2010) by the same authors.

8. D. Bernstein and P. Schwabe. New AES software speed records. INDOCRYPT 2008, LNCS vol. 5365, Springer, pp. 322-336, 2008.

9. A. Boldyreva and N. Taesombut. Online encryption schemes: new security notions and constructions. CT-RSA 2004, LNCS vol. 2964, Springer, pp. 1-14, 2004.

10. P. Fouque, A. Joux, G. Martinet, and F. Valette. Authenticated on-line encryption. SAC 2003, LNCS vol. 3006, Springer, pp. 145-159, 2003.

11. P. Fouque, A. Joux and G. Poupard. Blockwise adversarial model for on-line ciphers and symmetric encryption schemes. SAC 2004, LNCS vol. 3357, Springer, pp. 212-226, 2004.

12. P. Fouque, G. Martinet, G. Poupard. Practical symmetric on-line encryption. FSE 2003, LNCS vol. 2887, Springer, pp. 362-375, 2003.

13. S. Gueron and M. Kounavis. Intel carry-less multiplication instruction and its usage for computing the GCM mode (revision 2). White paper, available at www.intel.com. May 2010.

14. S. Halevi. EME*: extending EME to handle arbitrary-length messages with associated data. INDOCRYPT 2004, LNCS vol. 3348, Springer, pp. 315-327, 2004.

15. A. Joux, G. Martinet, and F. Valette. Blockwise-adaptive attackers: revisiting the (in)security of some provably secure encryption models: CBC, GEM, IACBC. CRYPTO 2002, LNCS vol. 2442, Springer, pp. 17-30, 2002.

16. H. Krawczyk. LFSR-based hashing and authentication. CRYPTO 1994, LNCS vol. 839, Springer, pp. 129-139, 1994.

17. M. Liskov, R. Rivest, and D. Wagner. Tweakable block ciphers. CRYPTO 2002, LNCS vol. 2442, Springer, pp. 31-46, 2002.

18. M. Luby and C. Rackoff. How to construct pseudorandom permutations from pseudorandom functions. SIAM Journal of Computing, 17(2), pp. 373-386, 1988.

19. D. McGrew and J. Viega. The security and performance of the Galois/counter mode (GCM) of operation. INDOCRYPT 2004, LNCS vol. 3348, Springer, pp. 343-355, 2004.

20. C. Meyer and M. Matyas. Cryptography: A New Dimension in Data Security. John Wiley \& Sons, New York, 1982.

21. M. Nandi. A simple security analysis of Hash-CBC and a new efficient one-key online cipher. Cryptology ePrint report 2007/158. May 7, 2007.

22. M. Nandi. Two New Efficient CCA-secure online ciphers: MHCBC and MCBC. INDOCRYPT 2008, LNCS vol. 5365, Springer, pp. 350-362, 2008. Also Cryptology ePrint report 2008/401, September 20, 2008.

23. M. Naor and O. Reingold. On the construction of pseudorandom permutations: Luby-Rackoff revisited. Journal of Cryptology, 12(1), pp. 29-66, 1999. 
24. T. Ristenpart and P. Rogaway. How to enrich the message space of a cipher. FSE 2007, LNCS vol. 4593, pp. 101-118, 2007. 